\title{
Subband architecture for Hybrid Filter Bank A/D converters
}

\author{
Davud Asemani, Member, IEEE, Jacques Oksman, and Pierre Duhamel, Fellow, IEEE
}

\begin{abstract}
Hybrid Filter Bank (HFB) A/D converters (ADC) are a candidate to realize the wide-band ADC for future telecommunication systems. The classical HFB-based ADCs are simulated in both time and frequency domains using simplyrealizable analog filters in this paper. The high sensitivity of HFB to realization errors of analysis filter bank is studied and it is shown that a new HFB architecture is necessary to employ the blind techniques for correcting the analog imperfections. Using the discrete-time model of HFB, a Multiple-Input MultipleOutput (MIMO) model is proposed for the HFB so that a new architecture called subband HFB-based ADC is obtained. The subband HFB ADC not only leads to a better resolution but is less sensitive to realization errors than the classical case. Besides, a Linear-Time Invariant (LTI) system represents the input-output relation of subband HFB without any spectral overlapping in contrary to the classical HFB. Thus, blind techniques may be used for correcting the realization errors in the subband HFB.
\end{abstract}

Index Terms-Hybrid Filter Bank, A/D converter, SoftwareDefined Radio.

\section{INTRODUCTION}

$\mathbf{T}$ $\mathrm{HE}$ demand for $\mathrm{A} / \mathrm{D}$ or $\mathrm{D} / \mathrm{A}$ converters with higher speeds has dramatically increased for realizing the new communications concepts such as Software-Defined Radio (SDR) approach [1]. Using a wide-band A/D or D/A converter, the conventional analog sharp filters and channelizer are substituted by digital filtering [2] and then the cost of receiver is independent of the channel number. Nowadays, the performance of ADCs can not still fulfill the requirements of the wide-band receiver of SDR approach. To achieve higher speeds of conversion, the use of $\mathrm{A} / \mathrm{D}$ converters in parallel has been attractive during the several decades [3], [4]. Time-interleaving and discrete-time Hybrid Filter Bank (HFB)A/D converters have been proposed to realize a parallel $A / D$ conversion [5], [6]. These structures include the on-chip compatibility with dense digital signal processors. The discrete-time Hybrid Filter Bank (HFB) architecture overcomes the problems of extremely high sensitivity to the mismatch of converters and timing errors from which time interleaving structure suffers much [7]. Considering the disadvantages of discrete-time filter bank such as limited band-width and switching noises, analog filter banks have been offered to operate instead of discretetime analysis filter bank in the HFB structures. Fig. 1 shows the continuous-time HFB-based A/D converter neglecting the quantization noise [8]. It includes two (analog analysis and digital synthesis) filter banks. Since the Perfect Reconstruction

First two authors (Firstname.Lastname@supelec.fr) are with the department of signal processing and electronic systems and the third one (Pierre.Duhamel@1ss.supelec.fr) is with the laboratory of signals and systems at École Supérieure d'Électricité (SUPELEC), Gif-sur-Yvette, 91192, France.

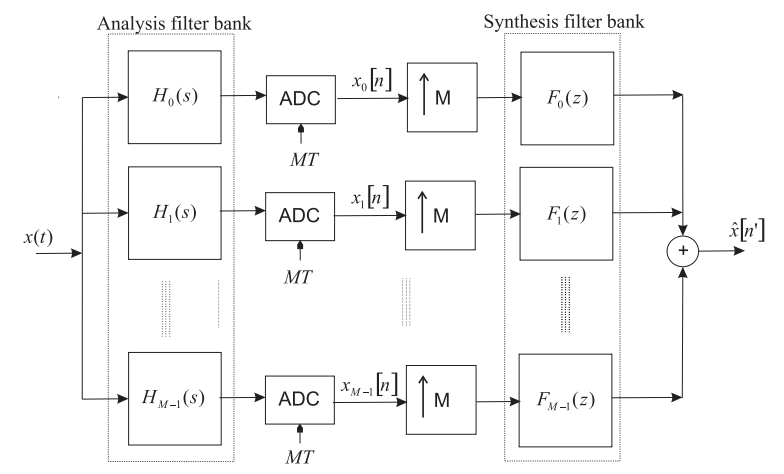

Fig. 1. The classical HFB-based A/D converter.

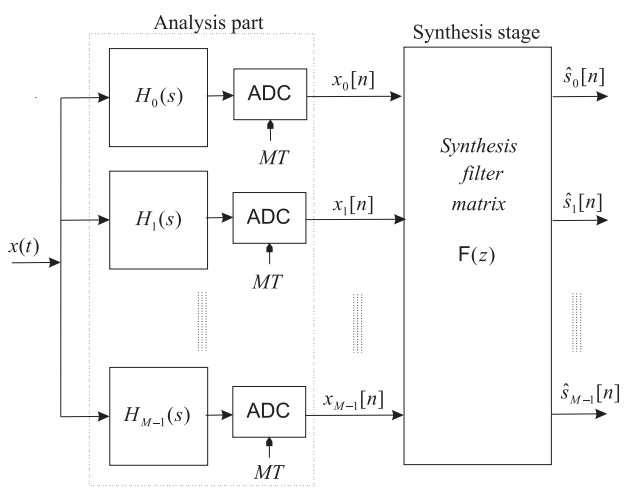

Fig. 2. The subband architecture of HFB-based A/D converter for estimating the subband components of the input signal.

(PR) condition can not hold in practice using Finite-Impulse Response (FIR) (or even Infinite-Impulse Response (IIR)) synthesis filters [9], [10], the reconstructed output $\hat{x}\left[n^{\prime}\right]$ of an $M$-branch HFB-based ADC is associated with the real input samples $x\left[n^{\prime}\right]=x\left(n^{\prime} T\right)$ through one distortion and $(M-1)$ interference (aliasing) terms [9]. To achieve a wide-band A/D conversion, the analog analysis filters are required to operate at high frequencies. Then, the feasibility of analog filters at high frequencies would be important. For this purpose, the simplyrealizable first- and second-order analog filters (equivalent to RC and RLC circuits) may be good candidates. Using these simple analysis filters along with simple FIR synthesis filters, an acceptable performance has been reported for HFBbased ADC if a small oversampling ratio is applied [11]. Different weighed sum of distortion and aliasing terms have been proposed as the optimization criterion for designing the HFB-based ADCs [12]. However, the fundamental challenge in the implementation of HFB-based A/D converters is the 
related high sensitivity to the realization errors [13]. In fact, a very small deviation in the parameters of analysis filter bank results in a large degradation of performance so that the respective HFB ADC would no longer be useful [14]. Digital compensation appears to be a suitable way to overcome this high sensitivity to realization errors of analog analysis filters. Sanada and Ikehara have proposed a decorrelating technique for correcting the realization errors [15]. Nevertheless, the proposed method needs a reference input to estimate the parameters of analysis filters and is not a blind technique (calibration). Besides, they have regarded a specific case where no synthesis filters are used. In this paper, the general HFB structures are considered in which simply-realizable first- and second-order analysis filters are used. The aim of this paper is to study firstly the origins of this high sensitivity to realization errors in the classical HFB-based ADCs. Secondly, a new architecture called subband HFB is proposed to which the methods such as blind deconvolution and decorrelation may be applied for correcting the realization errors without any need to the reference input (Fig. 2). To focus on the interference terms, quantization noises are neglected throughout this paper for convenience. This supposition may simply hold if a high resolution ADC is used at each branch of HFB so that the quantization noise is much less than the interference terms [7], [9]. In this case, the interference terms would be dominant for determining the output resolution. Throughout this paper, $n$ and $n^{\prime}$ are considered as the discrete-time indexes respectively associated with the sampling rates $\frac{1}{M T}$ and $\frac{1}{T}$ for better following the structures. In the next section, the classical HFB architecture is reviewed and studied. Then, the necessity of a new HFB architecture for correcting the analog non-idealities using the blind techniques is shown in section II. Section III provides a discrete-time model of HFB structure. Using the discrete-time model of HFB, subband HFB architecture is obtained and studied in section IV. At last, the results are summarized in the conclusion.

\section{The Classical HFB ADCs}

\section{A. Perfect Reconstruction Equations}

According to Fig. 1, the classical HFB-based ADC uses $M$ A/D converters sampling at the rate of $\frac{1}{M T}$ which is $M$ times less than the Nyquist rate $\frac{1}{T}$ associated with the analog input $x(t)$. The analog input signal $x(t)$ is supposed to be limited to the frequency interval $\left[\frac{-\pi}{T}, \frac{\pi}{T}\right]$. Realization of HFB A/D converter is feasible if and only if the presumed analysis filters hold some conditions [4]. This condition requires the analysis filters are chosen so that the respective analysis matrix is nonsingular at the band of interest. Neglecting quantization noise, the A/D converters at each branch of an $M$-branch HFB ADC (Fig. 1) may be replaced by a simple sampler at $\frac{1}{M T}$. Each signal $x_{k}[n](0 \leq k \leq M-1)$ may be obtained from filtering and then sampling the analog input $x(t)$. Then, the spectral representation $X_{k}\left(e^{j \omega}\right)$ of $x_{k}[n]$ can be described in terms of input $X(j \Omega)$ as:

$$
X_{k}\left(e^{j \omega}\right)=\frac{1}{M T} \sum_{p=-\infty}^{+\infty}\left[X(j \Omega) H_{k}(j \Omega)\right]_{\Omega=\frac{\omega}{M T}-\frac{2 \pi}{M T} p}
$$

where $\Omega$ and $\omega$ represent the frequency associated with the analog and discrete-time signals respectively. Then, the output $\widehat{x}\left[n^{\prime}\right]$ may be described in the frequency domain $\widehat{X}\left(e^{j \omega}\right)$ as following [9]:

$$
\widehat{X}\left(e^{j \omega}\right)=\frac{1}{M T} \sum_{p=-\infty}^{+\infty}\left[X(j \Omega) \cdot \sum_{k=0}^{M-1} H_{k}(j \Omega) \cdot F_{k}\left(e^{j \omega}\right)\right]_{\Omega=\frac{\omega}{T}-\frac{2 \pi}{M T} p}
$$

For better interpreting above relationship, $\widetilde{X}(j \Omega)$ and $\widetilde{H}_{k}(j \Omega)$ are supposed as the periodic extensions (with the period $\frac{2 \pi}{T}$ ) of $X(j \Omega)$ and $H_{k}(j \Omega)$ respectively limited to the frequency interval $\left[-\frac{\pi}{T}, \frac{\pi}{T}\right][16]$. Using $\widetilde{X}(j \Omega)$ and $\widetilde{H}_{k}(j \Omega)$, the output $\widehat{X}\left(e^{j \omega}\right)$ may be described as follows [16]:

$$
\widehat{X}\left(e^{j \omega}\right)=\frac{1}{M T} \sum_{p=0}^{M-1}\left[\widetilde{X}(j \Omega) \cdot \sum_{k=0}^{M-1} \widetilde{H}_{k}(j \Omega) \cdot F_{k}\left(e^{j \omega}\right)\right]_{\Omega=\frac{\omega}{T}-\frac{2 \pi}{M T} p}
$$

The equality (2) may be rewritten as follows:

$$
\widehat{X}\left(e^{j \omega}\right)=\underbrace{\tilde{X}\left(j \frac{\omega}{T}\right) \cdot T_{0}\left(e^{j \omega}\right)}_{\text {distortion part }}+\underbrace{\sum_{m=1}^{M-1} \widetilde{X}\left(j \frac{\omega}{T}-j \frac{2 \pi}{M T} m\right) \cdot T_{m}\left(e^{j \omega}\right)}_{\text {aliasing part }}
$$

where the distortion $T_{0}\left(e^{j \omega}\right)$ and aliasing $T_{m}\left(e^{j \omega}\right)$ functions $(m=1, \ldots, M-1)$ are:

$$
\left\{\begin{array}{l}
T_{0}\left(e^{j \omega}\right)=\frac{1}{M T} \sum_{k=0}^{M-1} F_{k}\left(e^{j \omega}\right) \cdot \widetilde{H}_{k}\left(j \frac{\omega}{T}\right) \\
T_{m}\left(e^{j \omega}\right)=\frac{1}{M T} \sum_{k=0}^{M-1} F_{k}\left(e^{j \omega}\right) \cdot \widetilde{H}_{k}\left(j \frac{\omega}{T}-j \frac{2 \pi}{M T} m\right)
\end{array}\right.
$$

The Perfect Reconstruction (PR) is accomplished when the output and input samples are the same except with a possible delay $n_{d}: \widehat{x}\left[n^{\prime}\right]=x\left(n^{\prime} T-n_{d} T\right)$. The PR conditions may be interpreted as the following equations:

$$
\left\{\begin{array}{l}
T_{0}\left(e^{j \omega}\right)=e^{-j \omega n_{d}} \\
T_{m}\left(e^{j \omega}\right)=0 \quad m=1, \ldots, M-1
\end{array}\right.
$$

\section{B. Designing the digital synthesis filters}

Using the PR equations (4), an HFB-based ADC may be designed provided that one of the respective synthesis or analysis filter banks are a priori known. Considering the constraints of analog circuits, it is practically preferred to design the digital synthesis filters according to a preselected set of analog circuits as analysis filters. To follow conveniently the design of digital synthesis filters, the PR equations are firstly described in the matrix-vectorial format. Invoking (4), the PR equations at the frequency $\omega$ may be described for an $M$-branch HFB-based ADC as follows:

$$
\mathbf{T}\left(e^{j \omega}\right)=\frac{1}{M T} \mathbf{H}\left(j \frac{\omega}{T}\right) \mathbf{F}\left(e^{j \omega}\right)
$$

where the vectors $\mathbf{T}\left(e^{j \omega}\right)$ and $\mathbf{F}\left(e^{j \omega}\right)(M \times 1)$ are:

$$
\begin{aligned}
\mathbf{T}\left(e^{j \omega}\right) & =\left[T_{0}\left(e^{j \omega}\right), T_{1}\left(e^{j \omega}\right), \cdots, T_{M-1}\left(e^{j \omega}\right)\right]^{T} \\
\mathbf{F}\left(e^{j \omega}\right) & =\left[F_{0}\left(e^{j \omega}\right), F_{1}\left(e^{j \omega}\right), \cdots, F_{M-1}\left(e^{j \omega}\right)\right]^{T}
\end{aligned}
$$


and $\mathbf{H}\left(j \frac{\omega}{T}\right)$ represents a matrix $(M \times M)$ whose $(m, n)^{t h}$ element is $\widetilde{H}_{n}\left(j \frac{\omega}{T}-j m \frac{2 \pi}{M T}\right) \quad(m, n \in[0, M-1])$. Assuming $N$ equally-spaced frequency points $\left\{\omega_{i}, \quad i=1,2, \cdots, N\right\}$, the equality (5) may be generalized in the matrix form as follows:

$$
\mathbf{T}_{N}=\frac{1}{M T} \mathbf{H}_{N} \mathbf{F}_{N}
$$

where the new vectors $\mathbf{T}_{N}$ and $\mathbf{F}_{N}(M N \times 1)$ are:

$$
\begin{aligned}
& \mathbf{T}_{N}=\left[\mathbf{T}\left(e^{j \omega_{1}}\right)^{T}, \mathbf{T}\left(e^{j \omega_{2}}\right)^{T}, \cdots, \mathbf{T}\left(e^{j \omega_{N}}\right)^{T}\right]^{T} \\
& \mathbf{F}_{N}=\left[\mathbf{F}\left(e^{j \omega_{1}}\right)^{T}, \mathbf{F}\left(e^{j \omega_{2}}\right)^{T}, \cdots, \mathbf{F}\left(e^{j \omega_{N}}\right)^{T}\right]^{T}
\end{aligned}
$$

and the analysis filters matrix $\mathbf{H}_{N}$ is as following:

$$
\mathbf{H}_{N}=\left[\begin{array}{cccc}
\mathbf{H}\left(j \frac{\omega_{1}}{T}\right) & & & \\
& \mathbf{H}\left(j \frac{\omega_{2}}{T}\right) & \mathbf{0} & \\
& \mathbf{0} & \ddots & \\
& & & \mathbf{H}\left(j \frac{\omega_{N}}{T}\right)
\end{array}\right]_{M N \times M N}
$$

Invoking (6), the PR equations may be established for the mentioned $N$ frequency points as following:

$$
\mathbf{H}_{N} \mathbf{F}_{N}=\mathbf{B}
$$

where the constant vector $\mathbf{B}$ is defined as follows:

$$
\begin{gathered}
\mathbf{B}=\left[B\left(e^{j \omega_{1}}\right)^{T}, B\left(e^{j \omega_{2}}\right)^{T}, \cdots, B\left(e^{j \omega_{N}}\right)^{T}\right]^{T} \\
B\left(e^{j \omega_{i}}\right)=M T\left[e^{-j \omega_{i} n_{d}}, 0, \cdots, 0\right]_{(M \times 1)}^{T}
\end{gathered}
$$

The frequency response of synthesis filters can be obtained from (7) using the known analysis filters. Assuming a known analysis filter bank, the problem is to design the respective suitable digital synthesis filters. The Finite-Impulse Response (FIR) filters are conveniently-realizable. Using FIR filters, the equations would be linear in terms of the unknown coefficients of synthesis filters as well. Considering IIR digital filters, the problem of instability may appear [18]. Supposing the FIR filters of the order $L-1$, the $k^{\text {th }}$ synthesis filter $f_{k}[n](k=$ $0,1, \cdots, M-1)$ is zero except for the range $0 \leq n \leq L-1$ as following:

$$
f_{k}=\left[f_{k}[0], f_{k}[1], \cdots, f_{k}[L-1]\right]^{T}
$$

$\mathbf{F}_{N}$ used in (7) may be related to the impulse responses $\left\{f_{k}[n], k=0,1, \cdots, M-1\right\}$ through the matrix $\mathbf{A}$ of Fourier transform as following:

$$
\mathbf{A . f}=\mathbf{F}_{N}
$$

where $\mathbf{f}$ is the overall vector of FIR synthesis filters as follows:

$$
\mathbf{f}=\left[f_{0}^{T}, f_{1}^{T}, \cdots, f_{M-1}^{T}\right]_{M L \times 1}^{T}
$$

and the matrix $\mathbf{A}$ of the Fourier transform is:

$$
\mathbf{A}=\left[\begin{array}{c}
A\left(e^{j \omega_{1}}\right) \\
A\left(e^{j \omega_{2}}\right) \\
\vdots \\
A\left(e^{j \omega_{N}}\right)
\end{array}\right]_{M N \times M L}
$$

that $A\left(e^{j \omega_{i}}\right)$ is itself another matrix as:

$$
A\left(e^{j \omega_{i}}\right)=\mathbf{I}_{M} \otimes a^{T}\left(e^{j \omega_{i}}\right)
$$

where $\mathbf{I}_{M}$ is the identity matrix $(M \times M)$ and $\otimes$ stands for the Kronecker production. The vector of $a^{T}\left(e^{j \omega_{i}}\right)$ is described as following:

$$
a^{T}\left(e^{j \omega_{i}}\right)=\left[1, e^{-j \omega_{i}}, \cdots, e^{-j \omega_{i}(L-1)}\right]_{1 \times L}
$$

Finally, according to the preceding relations, the impulse response $\mathbf{f}$ of synthesis filters may be related to the analysis filters matrix $\mathbf{H}$ as following:

$$
\mathbf{H}_{N} \mathbf{F}_{N}=\mathbf{B} \quad \mathbf{A f}=\mathbf{F}_{N}
$$

The first matrix equation in (9) (associated with (7)) consists of a square matrix $\mathbf{H}$ which has to be non-singular. The error emerges as soon as the second matrix equation in (9) (associated with the FIR approximation) is considered. The matrix $\mathbf{A}(M N \times M L)$ is necessarily chosen as a tall matrix $(N>L)$ to provide an acceptable interpolation. Then, the solution is not unique and can only approximate the associated equations. Integrating the relations of (9) and applying the Least Squares (LS) optimization technique, it yields:

$$
\begin{aligned}
\mathbf{f}_{\circ} & =\underset{\mathbf{f}}{\arg } \min \left\|\left(\mathbf{H}_{N} \mathbf{A}\right) \mathbf{f}-\mathbf{B}\right\|=\left(\mathbf{H}_{N} \mathbf{A}\right)^{\dagger} \mathbf{B} \\
& =\left[\left(\mathbf{H}_{N} \mathbf{A}\right)^{H}\left(\mathbf{H}_{N} \mathbf{A}\right)\right]^{-1}\left(\mathbf{H}_{N} \mathbf{A}\right)^{H} \mathbf{B}
\end{aligned}
$$

where $\mathbf{f}_{\circ}$ is the LS solution and $(.)^{\dagger}$ and $(.)^{H}$ represent the pseudo-inverse and conjugate-transpose of the operand respectively. Using $\mathbf{f}_{\circ}$, the ideal condition (the aliasing and distortion functions equal to zero and a pure delay system respectively) does not hold. The non-ideality depends on the optimization technique and $L$.

\section{Simulation of an eight-branch classical HFB ADC}

Using a simply-realizable class of analog circuits as the analysis filter bank, an eight-branch HFB-based A/D converter is designed and simulated in this section. It is supposed that the analysis filter bank is composed of the second-order RLC circuits except one first-order RC circuit as low-pass filter. All the second-order RLC circuits are assumed to have a constant passing band. The synthesis filters are supposed to be FIR digital filters with 64 coefficients. The simulations lead to a mean aliasing of $-46 d B$ which is not practically so acceptable (Fig. 3). When longer FIR synthesis filters are used, no important change is seen in the performance except a little improvement (the mean aliasing of $-53 d B$ for $L=128$ ). To improve the performance, it has been offered not 


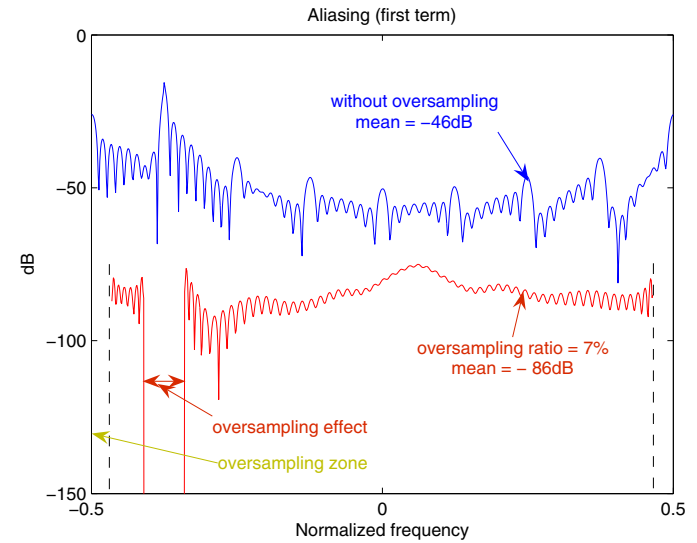

Fig. 3. First aliasing terms (in $d B$ ) vs. the normalized frequency for an eight-channel HFB structure. FIR synthesis filters have 64 coefficients and oversampling ratios are $7 \%$ and $0 \%$.

to consider the analog frequency borders (around $\pm \frac{\pi}{T}$ ) [11]. It would be equivalent to use a small oversampling ratio $\alpha$ so that the equations representing the border frequencies are eliminated because the input spectrum is now supposed to be $\left[-\frac{\pi}{T}(1-\alpha),+\frac{\pi}{T}(1-\alpha)\right]$ [11]. Using a small oversampling ratio, the preceding relationships (9) are still valid but a weighting matrix $\mathbf{W}$ is incorporated in (7). The weighting matrix $\mathbf{W}$ is an $M N \times M N$ identity matrix except some predefined diagonal elements are substituted by zero to eliminate the respective equation. The optimum oversampling ratio for this eight-branch HFB structure has been reported about 7\% [14]. The mean aliasing has been improved so much considering the oversampling ratio $\alpha=7 \%$. The distortion function is always acceptable $0 d B$ with the little variations $\left(<10^{-5} d B\right)$. For better comparing, the first aliasing terms are illustrated in Fig. 3 in the cases where the oversamling ratios of $7 \%$ and 0 are used. Fig. 3 shows a narrow band gap at the negative frequencies for the first aliaising term in the case of $\alpha=7 \%$. It represents the oversampling band with a shift of $m \frac{2 \pi}{M T}$ for each aliasing term $T_{m}\left(e^{j \omega}\right)$ according to (2) and (3) [16].

\section{NeCESSity OF A NEW ARCHITECTURE}

As it was explained in the previous section, the classical HFB-based A/D converter may provide a suitable performance by using the oversampling process. However, the analog circuits of analysis filter bank are in practice subject to the analog imperfections since the related electronic components include deviations from design values. The realization errors are mostly originated from the non-ideal phenomena due to fabrication [19]. Meanwhile, there are some time-dependent variations in the parameters of analog circuits as well. These analog imperfections may be associated with aging and ambience factors such as temperature drifts. The synthesis filter bank is designed according to the nominal parameters and not the real ones. So, the designed synthesis filter bank would not be optimum for the real analysis filter bank. On the other hand, HFB structures have exhibited a large sensitivity to the imperfections of analysis filter bank [14]. Considering the same eight-branch HFB structure of the previous subsection,
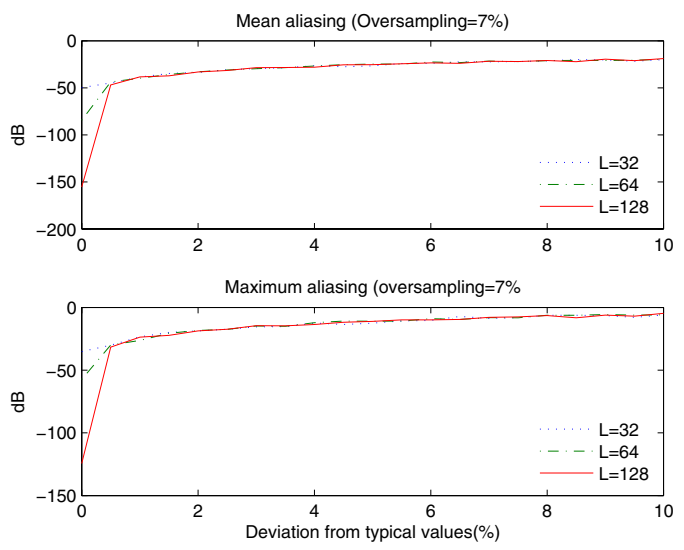

Fig. 4. Mean (above) and maximum (below) aliasing functions in $\mathrm{dB}$ versus the deviation from typical values (\%) for the the classical HFB ADC and the oversampling ratio $7 \%$. $L$ stands for the length of FIR synthesis filters.
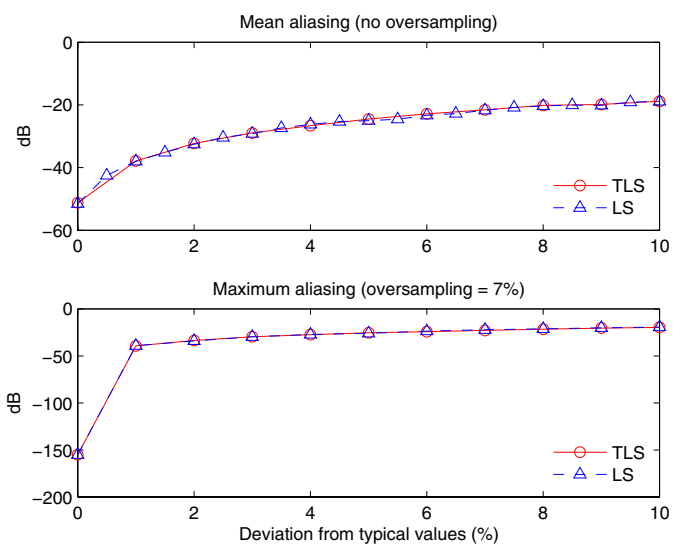

Fig. 5. Mean aliasing functions in $d B$ versus the deviation from typical values (\%) for TLS and LS optimization methods considering no oversampling (above) and the oversampling ratio 7\% (below) for $L=128$.

the simulations have been repeated for 1000 trials of the Gaussian realization errors. Figure 4 shows the mean and maximum values of aliasing function versus the deviations from nominal parameters of analysis filters considering the oversampling ratio of $7 \%$ for different lengths of FIR synthesis filters. The performance of classical HFB in presence of analog imperfections deteriorates largely [14] (Fig. 4). Total LeastSquares (TLS) optimization method is a candidate for decreasing the sensitivity to deviations of the coefficient matrix [20]. However, using the TLS, no improvement is obtained in the performance (Fig. 5). In fact, the TLS improves the performance for zero-residual problems [20]. The design of synthesis filter bank of HFB is not a zero-residual problem since there is no FIR synthesis filter bank leading to a null aliasing contribution (refer to the previous section). The sensitivity of solution (here synthesis filter bank) to the realization errors is proportional to the square of the condition number of coefficient matrix (here analysis matrix) [20]. Figure 6 exhibits the condition number of analysis matrix versus the oversampling ratio for the assumed eight-branch HFB. The condition number grows exponentially with the oversampling ratio. It means that 


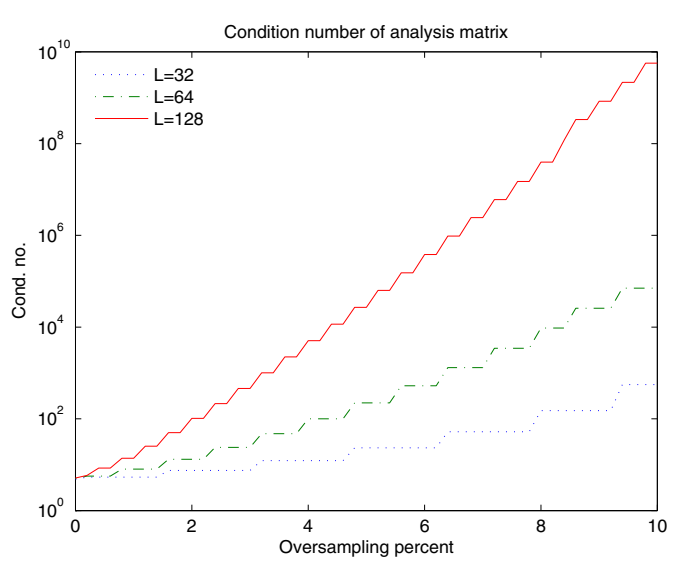

Fig. 6. The condition number of analysis matrix versus oversampling ratio (\%) for an eight-channel HFB A/D converter. $L$ represents the number of coefficients of FIR synthesis filters.

the oversampling process increases the sensitivity although it provides an improvement in the absence of realization errors. On the other hand, the classical HFB can not provide an acceptable performance unless the oversampling process is considered (section II-C). A modified classical HFB structure called two-stage HFB has been already proposed [16]. The two-stage HFB is even more sensitive to realization errors than the classical HFB though it provides an improvement in the absence of realization errors [14].

One of the possibilities to overcome this high sensitivity is to apply a compensation technique. Without calibration (nonblind) facilities, the required compensation method would use a blind estimation technique since neither input nor the exact transfer functions of analysis filters are known. If the realization errors are estimated, the proper transfer functions of analysis filters would be available for being used in the design of synthesis filters. Blind deconvolution method has already been used for estimating the realization errors of analog circuits [17]. For this purpose the included analog system has to be LTI as well as the Nyquist criterion is respected. This technique can not be applied to the output $x_{k}[n]$ of each branch in the HFB structure since there is an undersampling process included. It can not be applied to the global output $\widehat{x}[n]$ either since the HFB structure represents a non-LTI system in practice according to the aliasing terms. Then, it can not be directly applied to the classical HFB. Accordingly, a new architecture may be searched for HFBbased ADC to which blind techniques can be applied for estimating the realization errors. The new HFB architecture should represent an LTI system between the related inputoutput without any spectral overlapping.

\section{DISCRETE-TIME MODEL OF ANALYSIS FILTER}

For conveniently following the extraction of the new HFB structure (next section), it is better to have the analysis part fully described in the discrete-time domain. Thus, a discretetime model of analysis part is obtained in this subsection without loss of generality. The analysis part has been already modeled in the discrete-time domain by Shu et. al. to obtain

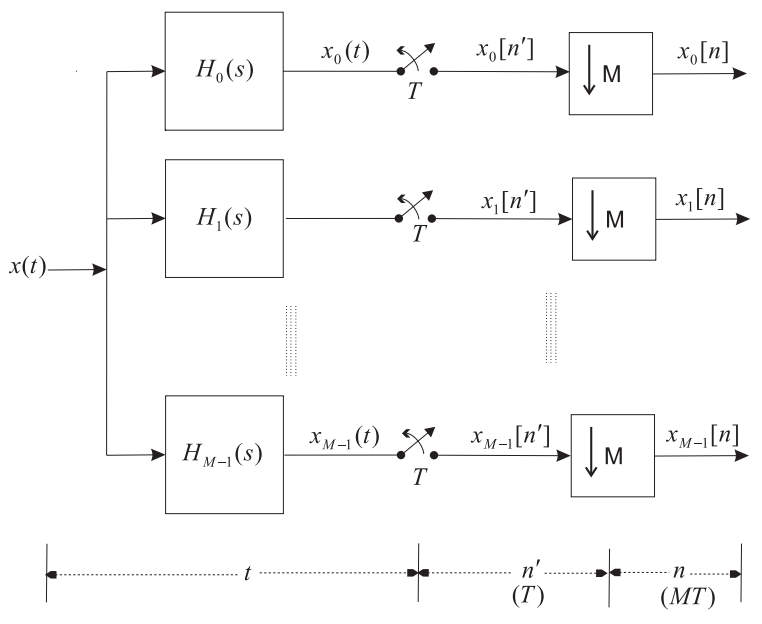

Fig. 7. The analysis part of the classical HFB-based A/D converter shown in terms of the decimation procedure.

a Minmax criterion [12]. We propose here a totally different method for providing the discrete-time model of analysis part. The analysis part of HFB is considered (see Fig. 2). Fig. 7 shows the analysis part where each A/D converter at $\frac{1}{M T}$ has been replaced with the tandem of a sampler at $\frac{1}{T}$ and a decimator (neglecting the quantization noise). The analog input $x(t)$ and the analysis filter bank are the only parameters which are not described in the discrete-time domain. The analog input $x(t)$ may be sampled without any spectral overlapping at $\frac{1}{T}$ since $x(t)$ holds the Nyquist rate $\frac{1}{T}$. Accordingly, $x(t)$ can be represented in the discrete-time domain by $x\left[n^{\prime}\right]$ where $x\left[n^{\prime}\right]=x\left(n^{\prime} T\right)$. Invoking Fig. 7, the output $x_{k}(t)$ of each analysis filter $H_{k}(s)$ can be explained in the frequency-domain as follows:

$$
X_{k}(j \Omega)=H_{k}(j \Omega) X(j \Omega) \quad k=0,2, \ldots, M-1
$$

$X_{k}(j \Omega)$ is band-limited since $X(j \Omega)$ is a band-limited signal (limited to the Nyquist rate $\frac{1}{T}$ ). Considering this property, $H_{k}(j \Omega)$ can be substituted with another analog filter $\check{H}_{k}(j \Omega)$ as follows:

$$
X_{k}(j \Omega)=\check{H}_{k}(j \Omega) X(j \Omega) \quad k \in\{0,2, \ldots, M-1\}
$$

where $\check{H}_{k}(j \Omega)$ is defined as:

$$
\check{H}_{k}(j \Omega)=\left\{\begin{array}{lr}
H_{k}(j \Omega) & \Omega \in\left[-\frac{\pi}{T},+\frac{\pi}{T}\right] \\
0 & \text { elsewhere }
\end{array}\right.
$$

According to (11), $\breve{H}_{k}(s)$ is evidently band-limited. Its impulse response $\breve{h}_{k}(t)$ may be sampled at $\frac{1}{T}$ without any spectral overlapping. If the continuous-time impulse response $\breve{h}_{k}(t)$ is sampled at the rate $\frac{1}{T}$, the discrete-time impulse response $h_{k}\left[n^{\prime}\right]$ may be obtained as following:

$$
h_{k}\left[n^{\prime}\right]=\breve{h}_{k}\left(n^{\prime} T\right) \quad n^{\prime}=\cdots,-2,-1,0,1,2,3, \cdots
$$

This relationship can be described in the frequency-domain as follows:

$$
H_{k}\left(e^{j \omega}\right)=\left.\frac{1}{T} \sum_{m=-\infty}^{+\infty} \check{H}_{k}\left(j \Omega-j \frac{2 \pi}{T} m\right)\right|_{\Omega=\frac{\omega}{T}}
$$




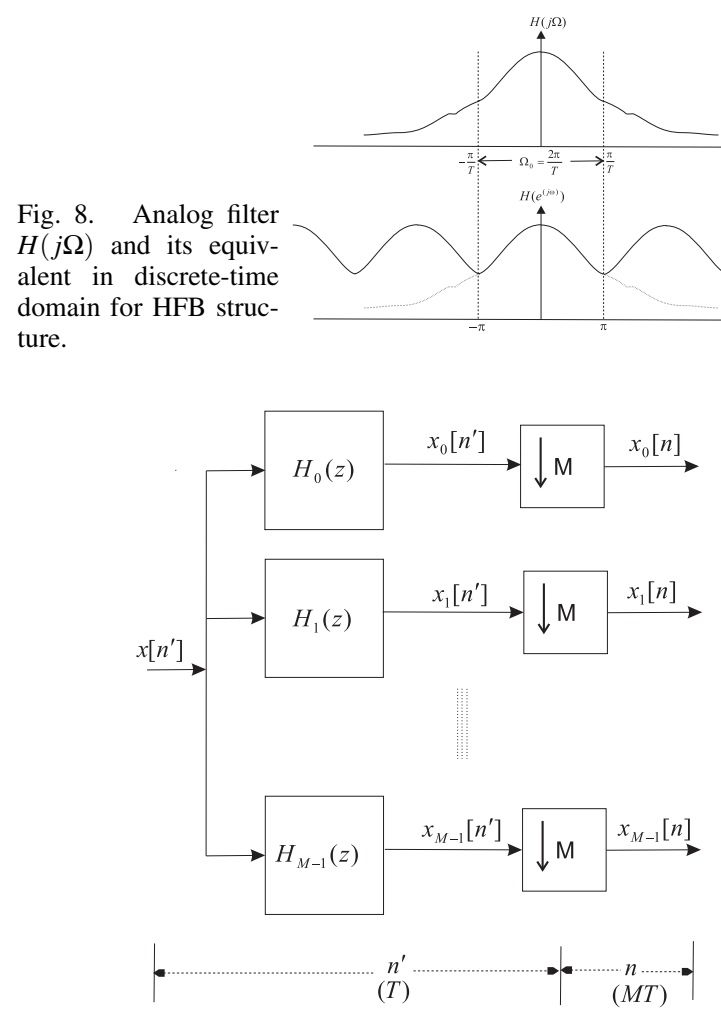

Fig. 9. The discrete-time model for the analysis part of HFB-based A/D converter. $n^{\prime}$ and $n$ represent the discrete-time indices associated with the sampling rates $\frac{1}{T}$ and $\frac{1}{M T}$ respectively.

Fig. 8 shows the discrete-time equivalent $H_{k}\left(e^{j \omega}\right)$ for an exemplary analog filter $H_{k}(j \Omega)$. Accordingly, the analog filter $H_{k}(j \Omega)$ can be substituted by $H_{k}\left(e^{j \omega}\right)$ in the discrete-time domain. Substituting $x(t)$ and $H_{k}(j \Omega)$ with $x\left[n^{\prime}\right]$ and $H_{k}\left(e^{j \omega}\right)$ respectively, the samplers are eliminated and the discrete-time model of analysis part is obtained. The related discrete-time model of analysis part is shown in Fig. 9. Considering that model, the objective of HFB-based A/D conversion is now to achieve the unknown signal $x\left[n^{\prime}\right]$.

\section{SUbBAND ARCHITECTURE FOR THE HFB-BASED ADCS}

\section{A. Simple illustration of Subband HFB concept}

Before mathematically extracting the (subband) MIMO model of HFB architecture, the principal idea is simply demonstrated in this subsection. For convenience, the analysis part of a two-branch HFB $(M=2)$ is considered. Without loss of generality, the frequency responses of the original input $X\left(e^{j \omega}\right)$ and the analysis filter $H_{0}\left(e^{j \omega}\right)$ are supposed to be as shown in Fig. 10. Invoking (1) and according to Fig. 9, $x_{0}[n]$ may be described as follows $(M=2)$ :

$$
X_{0}\left(e^{j \omega}\right)=\frac{1}{2} X\left(e^{j \frac{\omega}{2}}\right) \cdot H_{0}\left(e^{j \frac{\omega}{2}}\right)+\frac{1}{2} X\left(e^{j\left(\frac{\omega}{2}-\pi\right)}\right) \cdot H_{0}\left(e^{j\left(\frac{\omega}{2}-\pi\right)}\right)
$$

To better observe (12), this equality is shown in Fig. 10. The decimation procedure is carried out by two operations in Fig. 10 in reference with the two narrow-band parts (in bricks and hexagons): spectral dilating, and addition. If these two narrow-band components of the input signal are considered
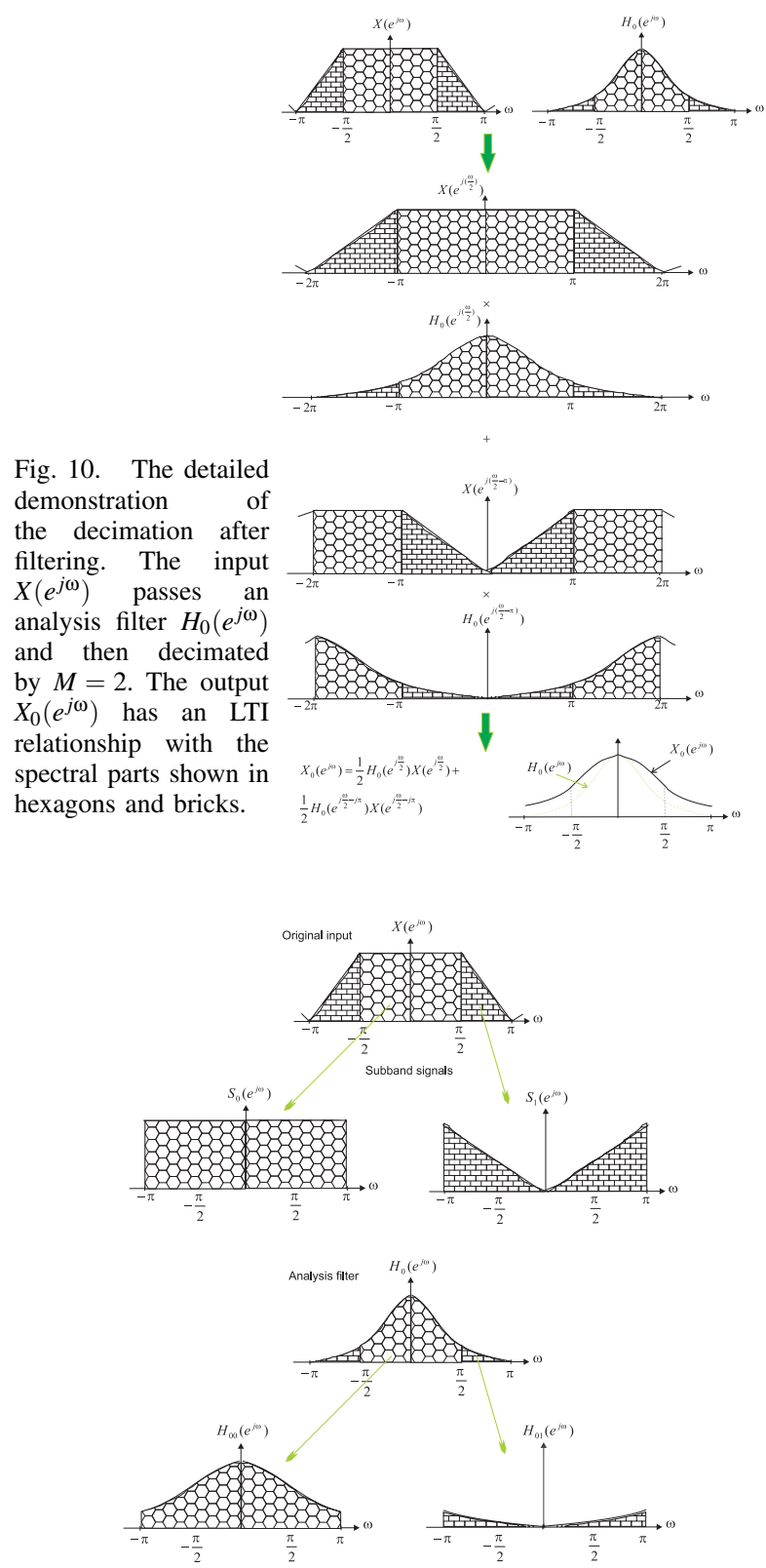

Fig. 11. The extraction of subband signals $S_{0}\left(e^{j \omega}\right)$ and $S_{1}\left(e^{j \omega}\right)$ from the original input $X\left(e^{j \omega}\right)$ for a two-branch HFB structure by decimating (one out of 2) the narrow-band (hexagonal and brick) parts. The associated LTI filters $H_{00}\left(e^{j \omega}\right)$ and $H_{01}\left(e^{j \omega}\right)$ may be obtained similarly from the analysis filter $H_{0}\left(e^{j \omega}\right)$.

as the virtual inputs, there exist no spectral overlapping and these new inputs would have an LTI relationship with the output $X_{0}\left(e^{j \omega}\right)$. Fig. 11 shows schematically these new input signals $S_{0}\left(e^{j \omega}\right)$ and $S_{1}\left(e^{j \omega}\right)$ obtained from the narrow-band components of $X\left(e^{j \omega}\right)$ by decimation. We call these signals the subband components of the input $x\left[n^{\prime}\right]$. It may be seen that $X_{0}\left(e^{j \omega}\right)$ is produced from the subband signals $S_{0}\left(e^{j \omega}\right)$ and $S_{1}\left(e^{j \omega}\right)$ as follows (Fig. 11):

$$
X_{0}\left(e^{j \omega}\right)=S_{0}\left(e^{j \omega}\right) H_{00}\left(e^{j \omega}\right)+S_{1}\left(e^{j \omega}\right) H_{01}\left(e^{j \omega}\right)
$$

According to (13), $X_{0}\left(e^{j \omega}\right)$ consists of the subband signals $S_{0}\left(e^{j \omega}\right)$ and $S_{1}\left(e^{j \omega}\right)$ passing through two LTI filters $H_{00}\left(e^{j \omega}\right)$ and $H_{01}\left(e^{j \omega}\right)$. In general case (Fig. 9), we can state that the 


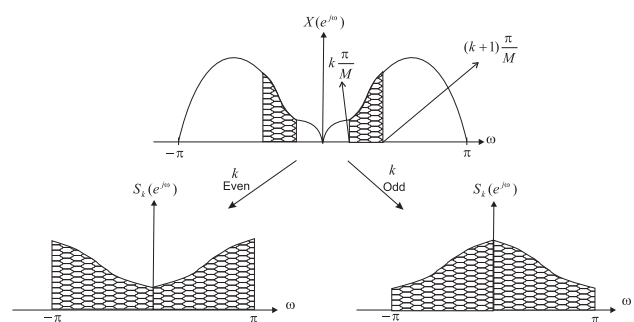

Fig. 12. The schematic illustration for extracting each subband signal $S_{k}\left(e^{j \omega}\right)$ $(0 \leq k \leq M-1)$ from the original signal $X\left(e^{j \omega}\right)$.

outputs $x_{0}[n], x_{1}[n], \ldots$, and $x_{M-1}[n]$ of analysis part may be associated to $M$ subband signals through an LTI relationship ( $M$ subband signals would exist for an $M$-branch HFB). Then, an LTI model may be established between virtual subband signals and outputs $x_{0}[n], x_{1}[n], \ldots$, and $x_{M-1}[n]$. This model is mathematically discussed and provided in the next subsection.

\section{B. Subband model of the HFB analysis part}

The discrete-time model of analysis part is considered (Fig. 9). It is proposed to consider $M$ discrete-time signals $s_{0}[n], s_{1}[n], \ldots$, and $s_{M-1}[n]$ called subband signals. Using the previous subsection V-A, each $s_{k}[n]$ may be extracted from the original input $x\left[n^{\prime}\right]$ in the frequency domain as shown in Fig. 12. When $k$ is even $(k=0,2, \ldots), S_{k}\left(e^{j \omega}\right)$ may mathematically be interpreted as follows (Fig. 12) [18]:

$$
S_{k}\left(e^{j \omega}\right)=\left\{\begin{array}{cr}
\frac{1}{M} X\left(e^{j \frac{\omega}{M}+j k \frac{\pi}{M}}\right) & \omega \in[0, \pi] \\
\frac{1}{M} X\left(e^{j \frac{\omega}{M}-j k \frac{\pi}{M}}\right) & \omega \in[-\pi, 0]
\end{array}\right.
$$

and for $k=1,3, \ldots$, it will be:

$$
S_{k}\left(e^{j \omega}\right)= \begin{cases}\frac{1}{M} X\left(e^{j \omega}-j(k+1) \frac{\pi}{M}\right) & \omega \in[0, \pi] \\ \frac{1}{M} X\left(e^{j \frac{\omega}{M}+j(k+1) \frac{\pi}{M}}\right) & \omega \in[-\pi, 0]\end{cases}
$$

The subband signals may be produced as shown in Fig. 13 where an ideal subband filter $G_{k}\left(e^{j \omega}\right)$ is used at each branch. $G_{k}\left(e^{j \omega}\right)$ is a virtual filter (no need to produce it) being zero at all the frequencies except $k \frac{\pi}{M} \leq|\omega| \leq(k+1) \frac{\pi}{M} \cdot G_{k}\left(e^{j \omega}\right)$ may be considered as demonstrated in Fig. 14. There is no spectral overlapping or ambiguity due to the decimation procedure in the production of $s_{0}[n], s_{1}[n], \ldots$, and $s_{M-1}[n]$ because of the narrow-band nature of subband filters.

The outputs of analysis part $\left(x_{0}[n], x_{1}[n], \ldots\right.$, and $\left.x_{M-1}[n]\right)$ can be reconstructed in terms of these subband signals. Similarly to (13), each $x_{k}[n]$ may be produced from the subband signals as following:

$$
X_{k}\left(e^{j \omega}\right)=\sum_{m=0}^{M-1} H_{k m}\left(e^{j \omega}\right) S_{k}\left(e^{j \omega}\right)
$$

where $H_{k 0}(z), H_{k 1}(z), \ldots$, and $H_{k(M-1)}(z)$ are extracted from the analysis filter $H_{k}(z)$ like to the subband signals (similar to (14) and (15)). Generalizing (16) to all the branches leads to the following matrix relation:

$$
\mathbf{x}[n]=\mathbf{H}[n] \star \mathbf{s}[n]
$$

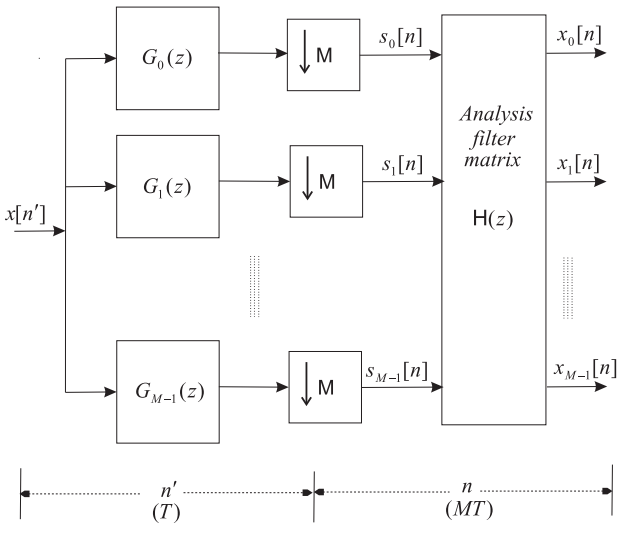

Fig. 13. The subband MIMO model for the analysis part of HFB-based A/D converter. The subband signals $\left\{s_{0}[n], s_{1}[n], \ldots, s_{M-1}[n]\right\}$ and $\left\{x_{0}[n], x_{1}[n], \ldots\right.$, and $\left.x_{M-1}[n]\right\}$ are inputs and outputs respectively.

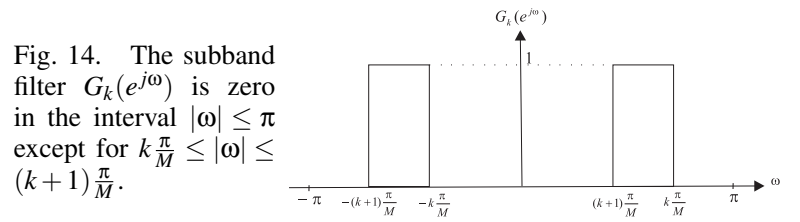

where $\star$ represents the element-wise convolution and the vectors $\mathbf{x}[n]$ and $\mathbf{s}[n]$ are:

$$
\begin{aligned}
& \mathbf{x}[n]=\left[x_{0}[n], x_{1}[n], \ldots, x_{M-1}[n]\right]^{T} \\
& \mathbf{s}[n]=\left[s_{0}[n], s_{1}[n], \ldots, s_{M-1}[n]\right]^{T}
\end{aligned}
$$

and $\mathbf{H}[n]$ is an $M \times M$ matrix of digital analysis filters and its $(k, m)^{t h}$ element is the impulse response of $H_{k m}(z)$. Accordingly, the vector of subband signals $\mathbf{s}[n]$ is related to the available signals vector $\mathbf{x}[n]$ through an LTI operation. Invoking (17) and using the preceding remarks, the analysis part of HFB structure may be modelled in the discretetime domain using a MIMO structure as shown in Fig. 13. Assuming the subband signals $\mathbf{s}[n]$ and the outputs of analysis part $\mathbf{x}[n]$ as the input and output vectors, an LTI MIMO system has been achieved. The decimation procedure exists no longer between input-output signals.

\section{Subband HFB-based ADC}

Using the MIMO model of analysis part (Fig. 13), a new HFB architecture for A/D conversion can be proposed. Figure 2 shows this subband HFB-based A/D converter applying a matrix of digital filters called synthesis filter matrix $\mathbf{F}(z)$ to the analysis part. This new ADC reconstructs the subband signals $s_{0}[n], s_{1}[n], \ldots$, and $s_{M-1}[n]$ instead of the original input $x\left[n^{\prime}\right]$ though $x\left[n^{\prime}\right]$ can be reconstructed through the computations from the subband signals. The synthesis filter matrix would tend to the inverse of analysis filter matrix $\mathbf{H}[n]$ used in (17). The MIMO model of analysis part (Fig. 13) may be employed for designing the synthesis filter matrix $\mathbf{F}(z)$. Considering 
Fig. 13 and Fig. 2, PR equations at each frequency $\omega$ will be:

$$
\mathbf{F}\left(e^{j \omega}\right) \cdot \mathbf{H}\left(e^{j \omega}\right)=\mathbf{I} \cdot e^{-j \omega n_{d}}
$$

where I represents the identity matrix $(M \times M)$ and $n_{d}$ stands for an arbitrary delay. $n_{d}$ is considered for maintaining the causality. $\mathbf{F}\left(e^{j \omega}\right)$ and $\mathbf{H}\left(e^{j \omega}\right)$ are the frequency representation of the synthesis and analysis filter matrices respectively. Solving (18) leads to the synthesis filters matrix $\mathbf{F}\left(e^{j \omega}\right)$ at each frequency $\omega$ as following:

$$
\mathbf{F}\left(e^{j \omega}\right)=e^{-j \omega n_{d}} \mathbf{H}^{-1}\left(e^{j \omega}\right)
$$

where the existence of $\mathbf{H}^{-1}\left(e^{j \omega}\right)$ has implicitly been assumed. The relation (19) may be established for $N$ equally-spaced frequency points (like to section II-B) so that the frequency response of each synthesis filter $F_{i j}\left(e^{j \omega}\right)$ can be achieved in the whole spectrum. Supposing that each synthesis filter is FIR with $L$ coefficients, $N$ must be selected larger than $L$ to have an overdetermined problem. On the other hand, the overfitting procedure may occur if $N$ is chosen much larger than $L$. In this paper, $N$ has been selected between $2 L$ and $3 L$. Using FIR synthesis filters, some distortion and interferences may appear at the output signals. Each output $\widehat{s}_{k}[n]$ can be interpreted in terms of distortion and Inter-Channel Interference (ICI) terms in this case. ICI terms are equivalent for the aliasing terms considered in the classical HFB structure (section II). Supposing the matrix $\mathbf{F}\left(e^{j \omega}\right)$ of FIR synthesis filters, $\mathbf{T}\left(e^{j \omega}\right)$ is defined as following:

$$
\mathbf{T}\left(e^{j \omega}\right)=\mathbf{F}\left(e^{j \omega}\right) \mathbf{H}\left(e^{j \omega}\right)
$$

$\mathbf{T}\left(e^{j \omega}\right)$ is a matrix containing distortion and ICI terms. It reveals that the estimated value $\widehat{s}_{k}[n]$ of $k^{\text {th }}$ subband signal $s_{k}[n]$ may be developed in the frequency domain as following:

$$
\widehat{S}_{k}\left(e^{j \omega}\right)=\underbrace{T_{k, k}\left(e^{j \omega}\right) S_{k}\left(e^{j \omega}\right)}_{\text {distortion term }}+\underbrace{\sum_{m=0, m \neq k}^{M-1} T_{k, m}\left(e^{j \omega}\right) S_{m}\left(e^{j \omega}\right)}_{\text {ICI terms }}
$$

The $(k)^{t h}$ diagonal element $T_{k k}\left(e^{j \omega}\right)$ of $\mathbf{T}\left(e^{j \omega}\right)$ stands for the distortion function related to the subband signal $S_{k}\left(e^{j \omega}\right)$. The other $M-1$ elements of $(k)^{t h}$ row of the $\mathbf{T}\left(e^{j \omega}\right)$ represent the relative ICI terms. $e^{-j \omega n_{d}}$ is the ideal value of the distortion function and the ICI elements are desired to be ideally null.

\section{Comparing subband and classical HFB architectures}

1) Performance with perfect analysis filters: An eightbranch subband HFB structure is considered using the same analysis filters of section II-C. The analysis filters are assumed to include no realization error. Supposing different lengths of FIR synthesis filters, the subband HFB-based ADC has been simulated in the frequency domain. The average ICI values are not so acceptable for practical applications reminding that the quantization noises are neglected (table I). The poor performance is related to the limitations of FIR digital filters used in the synthesis stage. For reducing the ICI terms, we offer to allocate a small part of frequency borders (low and high frequencies) at each subband spectrum as Guard Band (GB). Using a GB ratio of $7 \%$, the performance improves

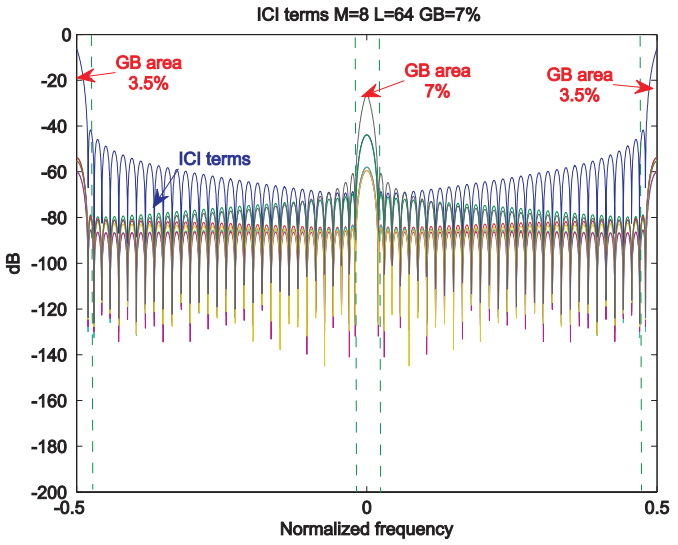

Fig. 15. The ICI terms due to the $4^{\text {th }}$ subband component versus normalized frequency. Each FIR synthesis filter includes 64 coefficients and the GB ratio is $7 \%$.

largely in terms of ICI functions (table I). The ICI terms associated with one of the subband signals $\left(s_{3}[n]\right)$ is illustrated in Fig. 15. Table I lists the ICI and distortion averages in $d B$ for the subband HFB structures in the cases of $0 \%$ and $7 \%$ guard band ratios. Larger GB ratios lead to a better performance in terms of the ICI terms because some constraints associated with the GB frequencies are relaxed in the design of FIR synthesis filters. On the other hand, changing GB has only slight effect on the sensitivity since the condition number of coefficient matrix remains unchanged. Using Simulink/Matlab, both the classical and subband HFB structures are simulated in the time domain to directly compare the related output resolutions with the same oversampling and GB ratios. A chirp signal covering the first subband spectrum is applied to both HFB structures as input. In the case of subband HFB, all subband outputs are null except the first subband $\widehat{s}_{0}[n]$ which is directly corresponding to the original input. Figure 16 shows the error spectra for the classical and subband HFBs. It may be seen that the error spectrum covers the whole spectrum in the classical HFB case though the input is limited to the first subband. Besides, a large signal appears at the oversampling spectral area which has to be filtered out. In the subband HFB case, this Post-Filtering (PF) procedure for filtering out the GB spectral area is not required unless the original input spectrum covers the GB parts. Using the well-known relation of $6 d B / b i t$ between SNR and resolution, the output resolution can be described in bits [18]. The output resolutions of both (subband and classical) HFB structures are listed in table II.

TABLE I

THE ICI AND DISTORTION AVERAGES OF AN EIGHT-BRANCH SUBBAND HFB STRUCTURE. $L$ REPRESENTS THE LENGTH OF FIR FILTERS.

\begin{tabular}{|c|c|c|c|c|}
\hline \multicolumn{5}{|c|}{ Eight-branch Subband HFB structure (in dB) } \\
\hline Guard band & \multicolumn{2}{|c|}{$0 \%$} & \multicolumn{2}{c|}{$7 \%$} \\
\hline$L$ & $\mathrm{ICl}$ mean & Distortion & $\mathrm{ICl}$ mean & Distortion \\
\hline 32 & -51 & -0.15 & -63 & $-7 \mathrm{E}-3$ \\
\hline 64 & -60 & -0.04 & -81 & $4.3 \mathrm{E}-4$ \\
\hline 128 & -67 & -0.02 & -118 & $2.2 \mathrm{E}-5$ \\
\hline
\end{tabular}




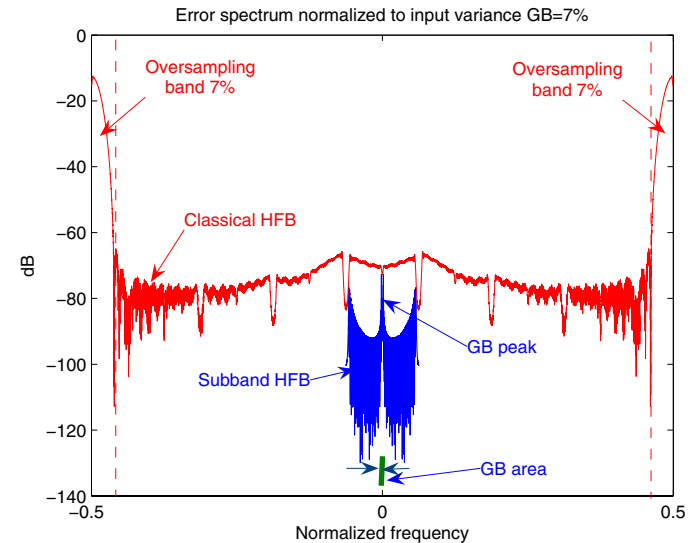

Fig. 16. The error spectra (in $\mathrm{dB}$ ) versus normalized frequency for the subband (blue) and classical (red) HFB structures. Considering the same oversampling and GB ratios of $7 \%$, each FIR synthesis filter includes 64 coefficients. A chirp sweeping the first subband is applied as the input signal.

It may be seen that the subband HFB-based ADC provides a slightly better resolution than the classical one supposing FIR synthesis filters of the same order.

2) Sensitivity to realization errors: To study the sensitivity to the realization errors, the mentioned eight-branch subband and classical HFB structures have been simulated considering Gaussian-distributed realization errors for the electronic elements of analysis filter bank. The simulations have been repeated for 1000 trials of random mutually-independent realization errors. Firstly, an analog input is considered including one sinusoidal signal located at the middle of first subband $\left(0.5 \frac{\pi}{8 T}\right)$. Fig. 17 shows the average resolution of both (subband and classical) HFB structures versus STD of error distribution considering 64 coefficients for each FIR synthesis filter. The resolutions have been shown in the presence or without $\mathrm{PF}$ procedure. As it was mentioned in the preceding subsection, $\mathrm{PF}$ process filters out the signal component at the oversampling and GB spectral areas for the classical and subband HFBs respectively. It is seen that the subband HFB exhibits less sensitive to the realization errors than the classical HFB for the sinusoidal input. The simulations have been repeated for a chirp input signal sweeping the first subband spectrum as well. Fig. 18 demonstrates the output resolutions versus the STD of error distribution. Again, the subband HFB appears less sensitive to the relation errors than the classical one. It may be seen the output resolution of classical HFB degrades so much if the PF process is not considered.

TABLE II

OUTPUT RESOLUTION OF CLASSICAL AND SUBBAND HFB-BASED ADCS. SUPPOSING THE SAME OVERSAMPLING AND GB RATIOS OF 7\%, FIR FILTERS WITH $L=64$ COEFFICIENTS ARE USED.

\begin{tabular}{|c|c|c|}
\hline \multicolumn{3}{|c|}{ Output resolution (in bits) } \\
\hline \multirow{2}{*}{ HFB architecture } & \multicolumn{2}{|c|}{ Input signal } \\
\cline { 2 - 3 } & Sinusoidal & Chirp \\
\hline Classical HFB & 9.9 & 9.6 \\
\hline Subband HFB & 10.5 & 10.1 \\
\hline
\end{tabular}

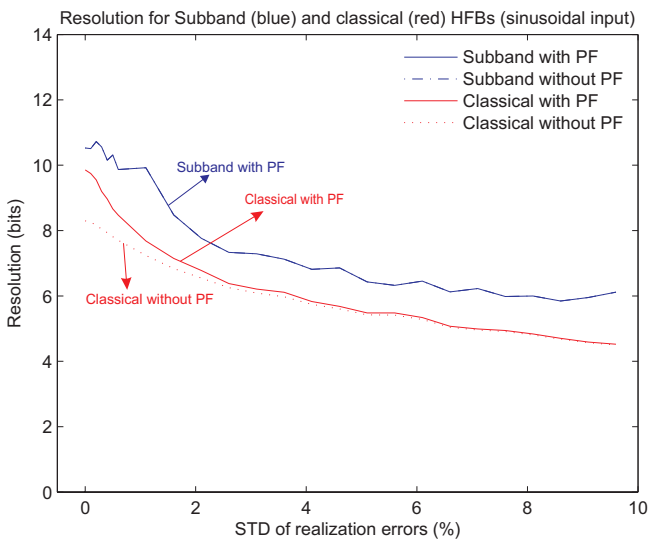

Fig. 17. The output resolution (in bits) of the classical and subband HFBbased ADCs versus STD of realization errors for a sinusoidal input. Each FIR synthesis filter includes 64 coefficients and both the oversampling and GB ratios are $7 \%$.

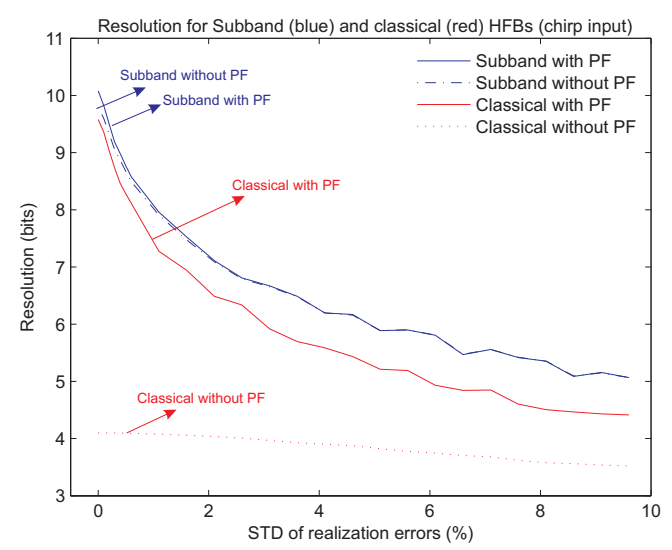

Fig. 18. The output resolution (in bits) of the classical and subband HFBbased ADCs versus STD of realization errors for a chirp input.

3) Computational complexity: The classical HFB-based A/D converter consists of $M$ FIR synthesis filters, but the subband architecture needs $M^{2}$ ones (compare Fig. 1 and Fig. 2). For one FIR filter with $L$ coefficients, $L$ multiplying operations and delay components are effectively necessary. Then, for implementing the synthesis stage, the subband architecture will need $M^{2} L$ multiplications to be compared with $M L$ ones in the classic case. Nevertheless, it does not require the upsampling operations (zero-padding by $M$ ) in the subband case. Moreover, the subband HFB structure provides $M$ output samples compared with only one output sample obtained from the classical HFB. Therefore, the computational complexity per each output sample is equivalent for both HFB structures.

To thoroughly compare the computational complexity, the design phase has to be considered as well. In the design phase, FIR synthesis filters are obtained. Assuming $N$ frequency points for designing the synthesis filters, conventional HFB structure would require the inversion of an $M N \times M N$ matrix. The subband HFB needs the inversion operation of $N$ matrices with the dimension $M \times M$. In practice, $N$ must be much 
larger than $M(N>>M)$ to have an acceptable interpolation (sections V-C and II-B). Thus, the design phase of classical HFB architecture is obviously much more complex than the subband one. The complexity of the design phase is particularly important when an adaptive method might be employed to estimate the real analysis filter bank for compensating realization errors.

\section{CONCLUSION}

The classical HFB-based ADC may provide an acceptable performance using perfect analog analysis filter bank provided that a small oversampling ratio is applied. The condition number of analysis filters matrix grows exponentially with the oversampling ratio as well as the related sensitivity to realization errors of analysis filters. The subband HFB-based may be used for directly A/D converting and demodulating the Frequency-Division Multiplexing (FDM) components of original analog input. The subband HFB provides at least a better performance of one bit compared to classical case. The subband HFB is less sensitive to the realization errors of analysis filters than the classical HFB as well. At last, the subband HFB can employ the blind techniques for correcting the analog imperfections. The subband HFB architecture seems interesting particularly when the information signals are accommodated in the FDM channels of a wide-band analog signal.

\section{REFERENCES}

[1] Joseph Mitola, Software Radios Survey, Critical Evaluation and Future Directions, IEEE AES Systems Magazine, Apr., 1983, pp. 25-36.

[2] B. Kraemer, Data conversion considerations for software radios, Proc.of IEEE Symp. on Spread Spectrum Techniques and Applications, vol. 2, Sept., 1998, pp. 546-550.

[3] A. Papoulis, Generalized Sampling Expansuion, IEEE Transaction On Circuits and Systems, vol.CAS-24, no.11, Nov., 1977.

[4] J. L. Brown, Multi-channel sampling of low pass signals, IEEE Transaction On Circuits and Systems, vol.CAS-28, no.2, Feb., 1981, pp. 101-106.

[5] W. C. Black and A. A. Hogges, Time interleaved converter arrays, IEEE Journal of Solid-state Circuits, vol.SC-15, no.6, Dec., 1980, pp. 10221029.

[6] A. Petraglia et al., QMF-Based A/D converters: Overview and new results, Proc. of Int. Conf. on A/D and D/A Conversion, Sept., 1991, pp. 112-117.

[7] S. R. Velazquez et al., Design of Hybrid Filter Banks for Analog/Digital conversion, IEEE Transactions On Signal Processing, vol.46, no.4, Apr., 1998, pp. 956-967. *

[8] Omid Oliaei, High-Speed $A / D$ and D/A converters using Hybrid Filter Banks, Proc. of IEEE Int. conf. on Electronics, Circuits and Systems, Sept., 1998, pp. 143-146.

[9] P. Lowenborg et al., Two-channel digital and hybrid Analog/Digital multirate filter banks with very low-complexity analysis or synthesis filters, IEEE Transactions on circuits and Systems, vol.50, no.7, July, 2003, pp. 355-367.

[10] C. Lelandais-Perrault et al., Hybrid filter bank A/D converters using IIR synthesis filters, IEEE Midwest Symposium on Circuits and Systems, Aug., 2005.

[11] T. Petrescu et al., Synthesis of hybrid filter banks by global frequency domain least squares solving, Proc. of IEEE Int. Symp. on Circuits And Systems, May, 2005.

[12] H. Shu et al., Minimax Design of Hybrid Multirate Filter Banks, IEEE Transactions on circuits and Systems-II: analog and digital signal processing, vol.44, no.2, Feb., 1997, pp. 120-128.

[13] P. Lowenborg and H. Johansson, Analysis of gain and timeskew errors in filter bank based A/D converters, Proc. of IEEE Int. Symp. on Circuits And Systems, June, 2001.
[14] D. Asemani and J. Oksman, Influences of oversampling and analog imperfections on hybrid filter bank $A / D$ converterss, Proc. of IEEE Int. Midwest Symp. on circuits And Systems, Aug., 2006.

[15] Y. Sanada and M. Ikehara, Decorrelating compensation scheme for coefficient errors of a filter bank parallel A/D converter, IEEE Transactions on wireless communications, vol.3, no.2, March, 2004, pp. 341-347.

[16] D. Asemani and J. Oksman, Two-stage synthesis filters for hybrid filter banks A/D converters, Proc. of IEEE Workshop on Signal Processing Systems, Nov., 2005, pp. 133-136.

[17] D. Asemani et al., Digital estimation of analog imperfections using blind equalization, Proc. of European Signal Processing Conference, Sept., 2006, Florence, Italy.

[18] S. K. Mitra, Digital Signal Processing: a computer-based approach, McGraw-Hill, 2001.

[19] Y. T Wang and A. A. Abidi, CMOS active filter design at very high frequencies, IEEE journal of solid-state circuits, vol.25, no.6, Dec., 1990 , pp. $1562-1574$

[20] S. V Huffel and J. Vanderwalle, The total Least Squares Problem: Computational Aspects and Analysis, SIAM, USA, 1991.

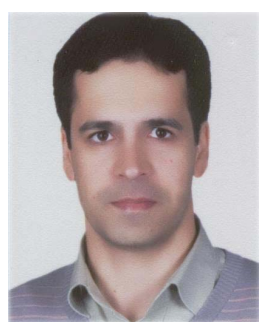

Davud Asemani (S'03-M'07) He received the B.Sc. and M.Sc. degrees in electronics and bioelectric engineering from Sharif University of Technology , Tehran, Iran in 1996 and 1998 resepectively and the Ph.D. degree in electronics engineering from SUPELEC, Gif-Sur-Yvette, France in 2007.

$\mathrm{He}$ is now with the department of electrical engineering of Tarbiat Modarres university, Tehran, Iran. His research interests include blind signal processing particularly applied to electronics systems and analog and digital electronic circuits design.

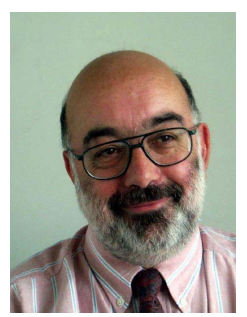

Jacques Oksman was born in Toulouse, France, in 1948. He received the "Diplôme d'Ingénieur" from the École Supérieure d'Électricité (SUPELEC) in 1971. He also holds an "Habilitation à diriger les recherches".

$\mathrm{He}$ is currently Professor at SUPELEC and Director of Research and Industry Partnership. His main interests are Signal Processing for measurement purposes and non-uniformly sampled signals. He has been working on various research projects involving such topics as parametric modeling of signals or systems for solving inverse problems or real-time identification or prediction of non-uniformly sampled signals. He teaches courses on Digital Design, Numerical Analysis and Optimization.

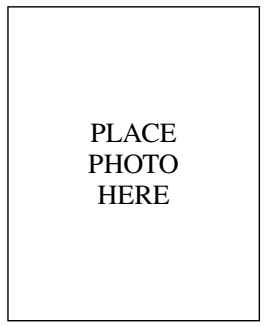

Pierre Duhamel Biography text here. 